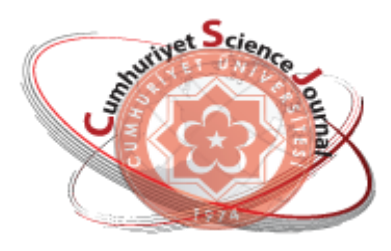

e-ISSN: $2587-246 X$

ISSN: $2587-2680$

\section{Cumanoryet Seience Journal $\cos$}

Cumhuriyet Sci. J., Vol.40-2 (2019) 355-368

\title{
On Solvability of An Inverse Boundary Value Problem For The Elliptic Equation Of Second Order With Periodic And Integral Condition
}

\author{
Amirov R. Kh. ${ }^{I^{*}(\mathrm{D})}$, Mehraliyev Y.T. ${ }^{2}$ (D), Heydarzade N.A ${ }^{2}$ (D) \\ ${ }^{I}$ Sivas Cumhuriyet University, Faculty of Science, Department of Mathematics Sivas , TURKEY, \\ ${ }^{2}$ Baku Statet University, Faculty of Mathematics, Baku, AZERBAYCAN
}

Received: 29.11.2018; Accepted: 19.03.2019

http://dx.doi.org/10.17776/csj.489939

\begin{abstract}
An inverse boundary value problem for a second-order elliptic equation with periodic and integral condition is investigated. The definition of a classical solution of the problem is introduced. The goal of this paper is to determine the unknown coefficient and to solve the problem of interest. The problem is considered in a rectangular domain. To investigate the solvability of the inverse problem, we perform a conversion from the original problem to some auxiliary inverse problem with trivial boundary conditions. By the contraction mapping principle we prove the existence and uniqueness of solutions of the auxiliary problem. Then we make a conversion to the stated problem again and, as a result, we obtain the solvability of the inverse problem.
\end{abstract}

Keywords: Inverse boundary value problem, Elliptic equation, Fourier method, Classical solution.

\section{İkinci Mertebeden Periyodik ve İntegral Koşullu Eliptik Denklemler İçin Ters Sınır-Değer Problemlerin Çözümü}

\begin{abstract}
Özet. Sunulan çalışmada dikdörtgensel bölgede periyodik ve integral tipli koşullarla verilen ikinci mertebeden eliptik tip diferansiyel denklemler için bazı ters problemlerin ç̧̈zümü ele alınmıştır. Matematiksel fiziğin denklemleri için ters problemler olarak verilen denklemlerin çözümü ile ilgili bilinen ek bilgiler yardımıyla onun katsayıların veya sağ taraftaki fonksiyonların, veya katsayıların ve sağ taraftaki bilinmeyen fonksiyonların birlikte belirlenmesi problemleri düşünülmektedir. Ters problemler bilimin birçok dallarında ortaya çıkmakta olup, özellikle fiziksel ve kimyasal süreçlerin takibi sırasında bazı büyüklüklerin belirlenmesinde önem taşımaktadır. Genelde fiziksel ve kimyasal süreçler diferansiyel denklemlerle ifade edildiğinden ve bu diferansiyel denklemlerin katsayıları da süreçleri ifade eden fiziksel ve kimyasal büyüklüklere bağlı olduğundan, süreçlerin akışının belirlenmesi için bu süreci ifade eden diferansiyel denklemin katsayılarının belirlenmesi önemlidir. Dolayısıyla, ters problemlerin konumu ve çözümü bilimsel açıdan çok önem taşımaktadır.
\end{abstract}

Anahtar Kelimeler: Ters sınır-değer problemleri,Elliptik denklem, Fourier yöntemi, Klasik çözüm

\section{INTRODUCTION}

In this paper, we discuss solutions of some inverse problems for second order elliptic differential equations under the periodic and integral type conditions in the rectangular area. By using well known additional information for the solution of inverse problems in mathematical physics equations, we think 
about the problem to determine their coefficients or right-hand side functions, or state coefficients and right-hand side unknown functions together. Inverse problems have wide-ranging applications in science, in particular, they are very important to determine some scalar quantities during the follow-up physical and chemical processes. In general, physical and chemical processes are expressed by differential equations and their coefficients depend on physical and chemical scalar quantities on which express their process, for these reasons, it is very important to determine these coefficients of differential equations to identify the flow of the process. Hence the position of inverse problem and their solutions are essential in science.

Among them we should mention the papers of A.N. Tikhonov [1], M.M. Lavrentyev [2, 3], V.K. Ivanov [4] and their followers. For a comprehensive overview, the reader should see the monograph by A.M. Denisov [5].

In this paper, we prove existence and uniqueness of the solution for the inverse boundary value problem for the elliptic equation of second order with periodic and integral type conditions.

\section{Problem statement and its reduction to an equivalent problem}

Consider the equation

$$
u_{t t}(x, t)+u_{x x}(x, t)=a(t) u(x, t)+b(t) g(x, t)+f(x, t)
$$

in the interior the domain $D_{T}=\{(x, t): 0 \leq x \leq 1,0 \leq t \leq T\}$ with the boundary conditions:

$$
u(x, 0)-\delta u_{t}(x, 0)=\varphi(x), u_{t}(x, T)=\psi(x)+\int_{0}^{T} p(t) u(x, t) d t \quad(0 \leq x \leq 1)
$$

periodic boundary condition

$$
u(0, t)=u(1, t) \quad(0 \leq t \leq T)
$$

nonlocal integral condition

$$
\int_{0}^{1} u(x, t) d x=0 \quad(0 \leq t \leq T)
$$

and the additional conditions

$$
u\left(x_{i}, t\right)=h_{i}(t) \quad\left(i=1,2 ; x_{1} \neq x_{2} ; 0 \leq t \leq T\right),
$$

where $\delta, x_{i} \in(0,1)(i=1,2)$-are fixed numbers, $f(x, t), g(x, t), \varphi(x), \psi(x), p(t), h(t)$-are given functions, $u(x, t), a(t)$ and $b(t)$ - are the unknown functions.

Definition . The triplet $\{u(x, t), a(t), b(t)\}$ is said to be a classical solution of problem (1)-(5), if the functions $u(x, t), a(t)$ and $b(t)$ satisfy the following conditions: 
1) The function $u(x, t)$ and its derivatives $u_{t}(x, t), u_{t t}(x, t), u_{x}(x, t), u_{x x}(x, t)$ are continuous in the domain $D_{T}$;

2) the functions $a(t)$ and $b(t)$ are continuous on the interval $[0, T]$;

3) equation (1) and conditions (2)-(5) are satisfied in the classical (usual) sense.

In order to investigate problem (1) - (5), we first consider the following auxiliary problem: it is required to define a triplet $\{u(x, t), a(t), b(t)\}$ of functions $u(x, t) \in C^{2}\left(D_{T}\right), a(t) \in C[0, T]$ and $b(t) \in C[0, T]$, from relations (1) - (3),

$$
\begin{gathered}
u_{x}(0 t)=u_{x}(1, t) \quad(0 \leq t \leq T), \\
h_{i}^{\prime \prime}(t)+u_{x x}\left(x_{i}, t\right)=a(t) h_{i}(t)+b(t) g\left(x_{i}, t\right)+f\left(x_{i}, t\right) \quad(i=1,2 ; 0 \leq t \leq T) .
\end{gathered}
$$

Analogously [6], the following lemma was proved.

Lemma1. Suppose that $\varphi(x), \psi(x) \in C[0,1], p(t) \in C[0, T], \quad h_{i}(t) \in C^{2}[0, T](i=1,2)$, $h(t) \equiv h_{1}(t) g\left(x_{2}, t\right)-h_{2}(t) g\left(x_{1}, t\right) \neq 0 \quad(0 \leq t \leq T), f(x, t) \in C\left(D_{T}\right), \int_{0}^{1} f(x, t) d x=0 \quad(0 \leq t \leq T)$ $, g(x, t) \in C\left(D_{T}\right), \quad \int_{0}^{1} g(x, t) d x=0 \quad(0 \leq t \leq T)$ and the compatibility conditions

$$
\begin{gathered}
\int_{0}^{1} \varphi(x) d x=0, \int_{0}^{1} \psi(x) d x=0, \\
\varphi\left(x_{i}\right)=h_{i}(0)-\delta h_{i}^{\prime}(0), \psi\left(x_{i}\right)=h_{i}^{\prime}(T)-\int_{0}^{T} p(t) h_{i}(t) d t(i=1,2) .
\end{gathered}
$$

hold. Then the following assertions are valid:

- each classical solution $\{u(x, t), a(t), b(t)\}$ of problem (1)-(5) is a solution of problem (1)-(3), (6), (7);

- each solution $\{u(x, t), a(t), b(t)\}$ of problem (1)-(3), (6), (7), satisfying the condition

$$
T\left((2 T+\delta)\|a(t)\|_{C[0, T]}+(T+\delta)\|p(t)\|_{C[0, T]}\right)<1,
$$

is a classical solution of problem (1)-(5).

\section{Existence and uniqueness of the classical solution to the inverse boundary value problem}

It is known [7] that the system 


$$
1, \cos \lambda_{1} x, \sin \lambda_{1} x, \ldots, \cos \lambda_{k} x, \sin \lambda_{k} x, \ldots
$$

is a basis in $L_{2}(0,1)$, where $\lambda_{k}=2 k \pi \quad(k=1, .2, .$.$) .$

Since the system (11) form a basis in $L_{2}(0,1)$, we shall seek the first component $u(x, t)$ of classical solution $\{u(x, t), a(t), b(t)\}$ of the problem (1)-(3), (6),(7) in the form

$$
u(x, t)=\sum_{k=0}^{\infty} u_{1 k}(t) \cos \lambda_{k} x+\sum_{k=1}^{\infty} u_{2 k}(t) \sin \lambda_{k} x \quad\left(\lambda_{k}=2 \pi k\right)
$$

where

$$
\begin{gathered}
u_{10}(t)=\int_{0}^{1} u(x, t) d x, \\
u_{1 k}(t)=2 \int_{0}^{1} u(x, t) \cos \lambda_{k} x d x \quad(k=1,2, \ldots), \\
u_{2 k}(t)=2 \int_{0}^{1} u(x, t) \sin \lambda_{k} x d x \quad(k=1,2, \ldots) .
\end{gathered}
$$

Then applying the formal scheme of the Fourier method, for determining of unknown coefficients $u_{1 k}(t) \quad(k=0,1, \ldots)$ and $u_{2 k}(t) \quad(k=1,2, \ldots)$ of function $u(x, t)$ from (1) and (2) we have

$$
\begin{gathered}
u_{10}^{\prime \prime}(t)=F_{10}(t ; u, a, b) \quad(0 \leq t \leq T), \\
u_{i k}^{\prime \prime}(t)-\lambda_{k}^{2} u_{i k}(t)=F_{i k}(t ; u, a, b) \quad(0 \leq t \leq T ; i=1,2 ; \quad k=1,2, \ldots), \\
u_{10}(0)-\delta u_{10}^{\prime}(0)=\varphi_{10}, u_{10}^{\prime}(T)=\psi_{10}+\int_{0}^{T} p(t) u_{10}(t) d t, \\
u_{i k}(0)-\delta u_{i k}^{\prime}(0)=\varphi_{i k}, u_{i k}^{\prime}(T)=\psi_{i k}+\int_{0}^{T} p(t) u_{i k}(t) d t(i=1,2 ; \quad k=1,2, \ldots),
\end{gathered}
$$

where

$$
F_{1 k}(t ; u, a, b)=a(t) u_{1 k}(t)+b(t) g_{1 k}(t)+f_{1 k}(t) \quad(k=0,1, \ldots)
$$


$g_{10}(t)=\int_{0}^{1} g(x, t) d x, \quad f_{10}(t)=\int_{0}^{1} f(x, t) d x, \quad \varphi_{10}=\int_{0}^{1} \varphi(x) d x, \quad \psi_{10}=\int_{0}^{1} \psi(x) d x$,

$g_{1 k}(t)=2 \int_{0}^{1} g(x, t) \cos \lambda_{k} x d x(k=1,2, \ldots), f_{1 k}(t)=2 \int_{0}^{1} f(x, t) \cos \lambda_{k} x d x(k=1,2, \ldots)$,

$\varphi_{1 k}=2 \int_{0}^{1} \varphi(x) \cos \lambda_{k} x d x, \quad \psi_{1 k}=2 \int_{0}^{1} \psi(x) \cos \lambda_{k} x d x \quad(k=1,2, \ldots)$,

$F_{2 k}(t ; u, a, b)=a(t) u_{2 k}(t)+b(t) g_{2 k}(t)+f_{2 k}(t)$,

$g_{2 k}(t)=2 \int_{0}^{1} g(x, t) \sin \lambda_{k} x d x(k=1,2, \ldots), f_{2 k}(t)=2 \int_{0}^{1} f(x, t) \sin \lambda_{k} x d x(k=1,2, \ldots)$,

$\varphi_{2 k}=2 \int_{0}^{1} \varphi(x) \sin \lambda_{k} x d x \quad, \quad \psi_{2 k}=2 \int_{0}^{1} \psi(x) \sin \lambda_{k} x d x \quad(k=1,2, \ldots)$.

Solving the problem (13)-(16), we find

$$
\begin{gathered}
u_{10}(t)=\varphi_{10}+\left(\psi_{10}+\int_{0}^{T} p(\tau) u_{10}(\tau) d t\right) t+\int_{0}^{T} G_{0}(t, \tau) F_{10}(\tau ; u, a, b) d \tau \\
u_{i k}(t)=\frac{\operatorname{ch}\left(\lambda_{k}(T-t)\right)}{\operatorname{ch}\left(\lambda_{k} T\right)+\lambda_{k} \delta \operatorname{sh}\left(\lambda_{k} T\right)} \varphi_{i k}+\frac{\operatorname{sh}\left(\lambda_{k} t\right)+\lambda_{k} \delta \operatorname{ch}\left(\lambda_{k} t\right)}{\lambda_{k}\left(\operatorname{ch}\left(\lambda_{k} T\right)+\lambda_{k} \delta \operatorname{sh}\left(\lambda_{k} T\right)\right)}\left(\psi_{i k}+\int_{0}^{T} p(\tau) u_{i k}(\tau) d t\right)+ \\
+\int_{0}^{T} G_{k}(t, \tau) F_{i k}(\tau ; u, a, b) d \tau \quad(i=1,2 ; k=1,2, \ldots)
\end{gathered}
$$

where

$$
\begin{gathered}
G_{0}(t, \tau)=\left\{\begin{array}{l}
-t-\delta, t \in[0, \tau] \\
-\tau-\delta, t \in[\tau, T],
\end{array}\right. \\
G_{k}(t, \tau)=\left\{\begin{array}{l}
\frac{\operatorname{ch}\left(\lambda_{k}(T-\tau)\right)}{\lambda_{k}\left(\operatorname{ch}\left(\lambda_{k} T\right)+\lambda_{k} \delta \operatorname{sh}\left(\lambda_{k} T\right)\right)}\left[\lambda_{k} \delta \operatorname{ch}\left(\lambda_{k} t\right)+\operatorname{sh}\left(\lambda_{k} t\right)\right], t \in[0, \tau], \\
\frac{\operatorname{ch}\left(\lambda_{k}(T-t)\right)}{\lambda_{k}\left(\operatorname{ch}\left(\lambda_{k} T\right)+\lambda_{k} \delta \operatorname{sh}\left(\lambda_{k} T\right)\right)}\left[\lambda_{k} \delta \operatorname{ch}\left(\lambda_{k} \tau\right)+\operatorname{sh}\left(\lambda_{k} \tau\right)\right], \quad t \in[\tau, T] .
\end{array}\right.
\end{gathered}
$$

After substituting the expressions $u_{1 k}(t)(k=0,1, \ldots)$ and $u_{2 k}(t)(k=1,2, \ldots)$ into (12), for the component $u(x, t)$ of the solution $\{u(x, t), a(t), b(t)\}$ to the problem (1)-(3), (6), (7) we get 


$$
\begin{aligned}
& u(x, t)=\varphi_{10}+\left(\psi_{10}+\int_{0}^{T} p(\tau) u_{10}(\tau) d t\right) t+\int_{0}^{T} G_{0}(t, \tau) F_{10}(\tau ; u, a, b) d \tau+ \\
& +\sum_{k=1}^{\infty}\left\{\frac{\operatorname{ch}\left(\lambda_{k}(T-t)\right)}{\operatorname{ch}\left(\lambda_{k} T\right)+\lambda_{k} \delta \operatorname{sh}\left(\lambda_{k} T\right)} \varphi_{1 k}+\frac{\operatorname{sh}\left(\lambda_{k} t\right)+\lambda_{k} \delta \operatorname{ch}\left(\lambda_{k} t\right)}{\lambda_{k}\left(\operatorname{ch}\left(\lambda_{k} T\right)+\lambda_{k} \delta \operatorname{sh}\left(\lambda_{k} T\right)\right)}\left(\psi_{1 k}+\int_{0}^{T} p(\tau) u_{1 k}(\tau) d t\right)+\right. \\
& \left.+\int_{0}^{T} G_{k}(t, \tau) F_{1 k}(\tau ; u, a, b) d \tau\right\} \cos \lambda_{k} x+ \\
& +\sum_{k=1}^{\infty}\left\{\frac{\operatorname{ch}\left(\lambda_{k}(T-t)\right)}{\operatorname{ch}\left(\lambda_{k} T\right)+\lambda_{k} \delta \operatorname{sh}\left(\lambda_{k} T\right)} \varphi_{2 k}+\frac{\operatorname{sh}\left(\lambda_{k} t\right)+\lambda_{k} \delta \operatorname{ch}\left(\lambda_{k} t\right)}{\lambda_{k}\left(\operatorname{ch}\left(\lambda_{k} T\right)+\lambda_{k} \delta \operatorname{sh}\left(\lambda_{k} T\right)\right)}\left(\psi_{2 k}+\int_{0}^{T} p(\tau) u_{2 k}(\tau) d t\right)+\right. \\
& \left.+\int_{0}^{T} G_{k}(t, \tau) F_{2 k}(\tau ; u, a, b) d \tau\right\} \sin \lambda_{k} x
\end{aligned}
$$

Now, using (7) and (12) we have

$$
\begin{gathered}
a(t)=[h(t)]^{-1}\left\{g\left(x_{2}, t\right)\left(h_{1}^{\prime \prime}(t)-f\left(x_{1}, t\right)\right)-g\left(x_{1}, t\right)\left(h_{2}^{\prime \prime}(t)-f\left(x_{2}, t\right)\right)-\right. \\
-\sum_{k=1}^{\infty} \lambda_{k}^{2} u_{1 k}(t)\left(g\left(x_{2}, t\right) \cos \lambda_{k} x_{1}-g\left(x_{1}, t\right) \cos \lambda_{k} x_{2}\right)- \\
\left.\quad-\sum_{k=1}^{\infty} \lambda_{k}^{2} u_{2 k}(t)\left(g\left(x_{2}, t\right) \sin \lambda_{k} x_{1}-g\left(x_{1}, t\right) \sin \lambda_{k} x_{2}\right)\right\}, \\
b(t)=[h(t)]^{-1}\left\{h_{1}(t)\left(h_{2}^{\prime \prime}(t)-f\left(x_{2}, t\right)\right)-h_{2}(t)\left(h_{1}^{\prime \prime}(t)-f\left(x_{1}, t\right)\right)-\right. \\
-\sum_{k=1}^{\infty} \lambda_{k}^{2} u_{1 k}(t)\left(h_{1}(t) \cos \lambda_{k} x_{2}-h_{2}(t) \cos \lambda_{k} x_{1}\right)- \\
\left.-\sum_{k=1}^{\infty} \lambda_{k}^{2} u_{2 k}(t)\left(h_{1}(t) \sin \lambda_{k} x_{2}-h_{2}(t) \sin \lambda_{k} x_{1}\right)\right\} .
\end{gathered}
$$

We substitute expression (18) into (20), (21) and have

$$
\begin{gathered}
a(t)=[h(t)]^{-1}\left\{g\left(x_{2}, t\right)\left(h_{1}^{\prime \prime}(t)-f\left(x_{1}, t\right)\right)-g\left(x_{1}, t\right)\left(h_{2}^{\prime \prime}(t)-f\left(x_{2}, t\right)\right)-\right. \\
-\sum_{k=1}^{\infty} \lambda_{k}^{2}\left[\frac{\operatorname{ch}\left(\lambda_{k}(T-t)\right)}{\operatorname{ch}\left(\lambda_{k} T\right)+\lambda_{k} \delta s h\left(\lambda_{k} T\right)} \varphi_{1 k}+\frac{\operatorname{sh}\left(\lambda_{k} t\right)+\lambda_{k} \delta \operatorname{ch}\left(\lambda_{k} t\right)}{\lambda_{k}\left(\operatorname{ch}\left(\lambda_{k} T\right)+\lambda_{k} \delta s h\left(\lambda_{k} T\right)\right)}\left(\psi_{1 k}+\int_{0}^{T} p(\tau) u_{1 k}(\tau) d t\right)-\right.
\end{gathered}
$$




$$
\begin{aligned}
& \left.+\int_{0}^{T} G_{k}(t, \tau) F_{1 k}(\tau ; u, a, b) d \tau\right]\left(g\left(x_{2}, t\right) \cos \lambda_{k} x_{1}-g\left(x_{1}, t\right) \cos \lambda_{k} x_{2}\right)- \\
& -\sum_{k=1}^{\infty} \lambda_{k}^{2}\left[\frac{\operatorname{ch}\left(\lambda_{k}(T-t)\right)}{\operatorname{ch}\left(\lambda_{k} T\right)+\lambda_{k} \delta \operatorname{sh}\left(\lambda_{k} T\right)} \varphi_{2 k}+\frac{\operatorname{sh}\left(\lambda_{k} t\right)+\lambda_{k} \delta \operatorname{ch}\left(\lambda_{k} t\right)}{\lambda_{k}\left(\operatorname{ch}\left(\lambda_{k} T\right)+\lambda_{k} \delta s h\left(\lambda_{k} T\right)\right.}\left(\psi_{2 k}+\int_{0}^{T} p(\tau) u_{2 k}(\tau) d t\right)-\right. \\
& \left.\left.+\int_{0}^{T} G_{k}(t, \tau) F_{2 k}(\tau ; u, a, b) d \tau\right]\left(g\left(x_{2}, t\right) \sin \lambda_{k} x_{1}-g\left(x_{1}, t\right) \sin \lambda_{k} x_{2}\right)\right\}, \\
& b(t)=[h(t)]^{-1}\left\{h_{1}(t)\left(h_{2}^{\prime \prime}(t)-f\left(x_{2}, t\right)\right)-h_{2}(t)\left(h_{1}^{\prime \prime}(t)-f\left(x_{1}, t\right)\right)-\right. \\
& -\sum_{k=1}^{\infty} \lambda_{k}^{2}\left[\frac{\operatorname{ch}\left(\lambda_{k}(T-t)\right)}{\operatorname{ch}\left(\lambda_{k} T\right)+\lambda_{k} \delta \operatorname{sh}\left(\lambda_{k} T\right)} \varphi_{1 k}+\frac{\operatorname{sh}\left(\lambda_{k} t\right)+\lambda_{k} \delta \operatorname{ch}\left(\lambda_{k} t\right)}{\lambda_{k}\left(\operatorname{ch}\left(\lambda_{k} T\right)+\lambda_{k} \delta s h\left(\lambda_{k} T\right)\right)}\left(\psi_{1 k}+\int_{0}^{T} p(\tau) u_{1 k}(\tau) d t\right)-\right. \\
& \left.+\int_{0}^{T} G_{k}(t, \tau) F_{1 k}(\tau ; u, a, b) d \tau\right]\left(h_{1}(t) \cos \lambda_{k} x_{2}-h_{2}(t) \cos \lambda_{k} x_{1}\right)- \\
& -\sum_{k=1}^{\infty} \lambda_{k}^{2}\left[\frac{\operatorname{ch}\left(\lambda_{k}(T-t)\right)}{\operatorname{ch}\left(\lambda_{k} T\right)+\lambda_{k} \delta \operatorname{sh}\left(\lambda_{k} T\right)} \varphi_{2 k}+\frac{\operatorname{sh}\left(\lambda_{k} t\right)+\lambda_{k} \delta \operatorname{ch}\left(\lambda_{k} t\right)}{\lambda_{k}\left(\operatorname{ch}\left(\lambda_{k} T\right)+\lambda_{k} \delta \operatorname{sh}\left(\lambda_{k} T\right)\right)}\left(\psi_{2 k}+\int_{0}^{T} p(\tau) u_{2 k}(\tau) d t\right)-\right. \\
& \left.\left.+\int_{0}^{T} G_{k}(t, \tau) F_{2 k}(\tau ; u, a, b) d \tau\right]\left(h_{1}(t) \sin \lambda_{k} x_{2}-h_{2}(t) \sin \lambda_{k} x_{1}\right)\right\},
\end{aligned}
$$

Thus, the problem (1)-(3), (6), (7) is reduced to solving the system (19), (22), (23) with respect to the unknown functions $u(x, t), a(t)$ and $b(t)$.

Using the definition of the solution of the problem (1)-(3), (6),(7), we prove the following lemma.

Lemma 2. If $\{u(x, t), a(t), b(t)\}$ is any solution to the problem (1)-(3), (6), (7), then the functions

$$
\begin{gathered}
u_{10}(t)=\int_{0}^{1} u(x, t) d x, \\
u_{1 k}(t)=2 \int_{0}^{1} u(x, t) \cos \lambda_{k} x d x \quad(k=1,2, \ldots),
\end{gathered}
$$




$$
u_{2 k}(t)=2 \int_{0}^{1} u(x, t) \sin \lambda_{k} x d x \quad(k=1,2, \ldots)
$$

satisfy the system (17), (18) in $[0, T]$.

Remark. It follows from lemma 2 that to prove the uniqueness of the solution to the problem (1)-(3), (6), (7), it suffices to prove the uniqueness of the solution to the system (19), (22) , (23)

. In order to investigate the problem (1) - (3), (6), (7), consider the following spaces

Denote by $B_{2, T}^{3}[6]$ the set of all functions $u(x, t)$ of the form

$$
u(x, t)=\sum_{k=0}^{\infty} u_{1 k}(t) \cos \lambda_{k} x+\sum_{k=1}^{\infty} u_{2 k}(t) \sin \lambda_{k} x \quad(\lambda=2 k \pi)
$$

defined on $D_{T}$ such that the functions $u_{1 k}(t) \quad(k=0,1,2, \ldots), u_{2 k}(t) \quad(k=1,2, \ldots)$ are continuous on $[0, T]$ and

$$
\left\|u_{10}(t)\right\|_{C[0, T]}+\left(\sum_{k=1}^{\infty}\left(\lambda_{k}^{3}\left\|u_{1 k}(t)\right\|_{C[0, T]}\right)^{2}\right)^{\frac{1}{2}}+\left(\sum_{k=1}^{\infty}\left(\lambda_{k}^{3}\left\|u_{2 k}(t)\right\|_{C[0, T]}\right)^{2}\right)^{\frac{1}{2}}<+\infty
$$

The norm on this set is given by

$$
\|u(x, t)\|_{B_{2, T}^{3}}=\left\|u_{10}(t)\right\|_{C[0, T]}+\left(\sum_{k=1}^{\infty}\left(\lambda_{k}^{3}\left\|u_{1 k}(t)\right\|_{C[0, T]}\right)^{2}\right)^{\frac{1}{2}}+\left(\sum_{k=1}^{\infty}\left(\lambda_{k}^{3}\left\|u_{2 k}(t)\right\|_{C[0, T]}\right)^{2}\right)^{\frac{1}{2}} .
$$

Denote by $E_{T}^{3}$ the space $\left.B_{2, T}^{3} \times C[0, T]\right]$ of the vector-functions $z(x, t)=\{u(x, t), a(t), b(t)\}$ with the norm

$$
\|z(x, t)\|_{B_{2, T}^{3}}=\|u(x, t)\|_{B_{2, T}^{3}}+\|a(t)\|_{C[0, T]}+\|b(t)\|_{C[0, T]} .
$$

It is known that $B_{2, T}^{3}$ and $E_{T}^{3}$ are Banach spaces.

Now, in the space $E_{T}^{3}$ consider the operator

$$
\Phi(u, a, b)=\left\{\Phi_{1}(u, a, b), \Phi_{2}(u, a, b), \Phi_{3}(u, a, b)\right\}
$$

where

$\Phi_{1}(u, a, b)=\tilde{u}(x, t) \equiv \sum_{k=0}^{\infty} \tilde{u}_{1 k}(t) \cos \lambda_{k} x+\sum_{k=1}^{\infty} \tilde{u}_{2 k}(t) \sin \lambda_{k} x, \Phi_{2}(u, a, b)=\tilde{a}(t), \quad \Phi_{3}(u, a, b)=\tilde{b}(t), \mathrm{a}$ $\tilde{u}_{10}(t), \tilde{u}_{i k}(t) \quad(i=1,2 ; k=0,1,2, \ldots), \tilde{a}(t)$ and $\tilde{b}(t)$ equal to the right hand sides of (17), (18), (22) 
and (23), respectively.

It is easy to see that

$$
\begin{gathered}
\frac{\operatorname{ch}\left(\lambda_{k}(T-t)\right)}{\operatorname{ch}\left(\lambda_{k} T\right)+\lambda_{k} \delta \operatorname{sh}\left(\lambda_{k} T\right)}<1 \quad(0 \leq t \leq T), \frac{\operatorname{sh}\left(\lambda_{k} t\right)+\lambda_{k} \delta \operatorname{ch}\left(\lambda_{k} t\right)}{\lambda_{k}\left(\operatorname{ch}\left(\lambda_{k} T\right)+\lambda_{k} \delta \operatorname{sh}\left(\lambda_{k} T\right)\right)}<1+\delta \quad(0 \leq t \leq T), \\
\frac{\operatorname{ch}\left(\lambda_{k}(T-\tau)\right)}{\lambda_{k}\left(\operatorname{ch}\left(\lambda_{k} T\right)+\lambda_{k} \delta \operatorname{sh}\left(\lambda_{k} T\right)\right)}\left[\lambda_{k} \delta \operatorname{ch}\left(\lambda_{k} t\right)+\operatorname{sh}\left(\lambda_{k} t\right)\right]<(1+\delta) \quad(0 \leq t \leq \tau \leq T), \\
\frac{\operatorname{ch}\left(\lambda_{k}(T-t)\right)}{\lambda_{k}\left(\operatorname{ch}\left(\lambda_{k} T\right)+\lambda_{k} \delta \operatorname{sh}\left(\lambda_{k} T\right)\right)}\left[\lambda_{k} \delta \operatorname{ch}\left(\lambda_{k} \tau\right)+\operatorname{sh}\left(\lambda_{k} \tau\right)\right]<(1+\delta) \quad(0 \leq \tau \leq t \leq T) .
\end{gathered}
$$

Taking into account these relations, with the help of simple transformations we find:

$$
\begin{aligned}
& \left\|\tilde{u}_{10}(t)\right\|_{C[0, T]} \leq\left|\varphi_{10}\right|+T\left(\left|\psi_{10}\right|+T\|p(t)\|_{C[0, T]}\left\|u_{10}(t)\right\|_{C[0, T]}\right)+\left[\sqrt{T}\left(\int_{0}^{T}\left|f_{10}(\tau)\right|^{2} d \tau\right)^{\frac{1}{2}}+\right. \\
& \left.+T\|a(t)\|_{C[0, T]}\left\|u_{10}(t)\right\|_{C[0, T]}+\sqrt{T}\left(\int_{0}^{T}\left|g_{10}(\tau)\right|^{2} d \tau\right)^{\frac{1}{2}}\|b(t)\|_{C[0, T]}\right](T+\delta), \\
& \left(\sum_{k=1}^{\infty}\left(\lambda_{k}^{3}\left\|\tilde{u}_{i k}(t)\right\|_{C[0, T]}\right)^{2}\right)^{\frac{1}{2}} \leq \sqrt{6}\left(\sum_{k=1}^{\infty}\left(\lambda_{k}^{3}\left|\varphi_{i k}\right|\right)^{2}\right)^{\frac{1}{2}}+ \\
& +\sqrt{6}(1+\delta)\left[\left(\sum_{k=1}^{\infty}\left(\lambda_{k}^{3}\left|\psi_{i k}\right|\right)^{2}\right)^{\frac{1}{2}}+T\|p(t)\|_{C[0, T]}\left(\sum_{k=1}^{\infty}\left(\lambda_{k}^{3}\left\|u_{i k}(t)\right\|_{C[0, T]}\right)^{2}\right)^{\frac{1}{2}}\right]+ \\
& +\sqrt{6}(1+\delta)\left[\sqrt{T}\left(\int_{0}^{T} \sum_{k=1}^{\infty}\left(\lambda_{k}^{3}\left|f_{i k}(\tau)\right|\right)^{2}\right)^{\frac{1}{2}}+T\|a(t)\|_{C[0, T]}\left(\sum_{k=1}^{\infty}\left(\lambda_{k}^{3}\left\|u_{i k}(t)\right\|_{C[0, T]}\right)^{2}\right)^{\frac{1}{2}}+\right. \\
& \left.+\sqrt{T}\left(\int_{0}^{T} \sum_{k=1}^{\infty}\left(\lambda_{k}^{3}\left|g_{i k}(\tau)\right|\right)^{2}\right)^{\frac{1}{2}}\|b(t)\|_{C[0, T]}\right]
\end{aligned}
$$

$\|\tilde{a}(t)\|_{C[0, T]} \leq\left\|[h(t)]^{-1}\right\|_{C[0, T]}\left\{\left\|g\left(x_{1}, t\right)\left(h_{1}^{\prime \prime}(t)-f\left(x_{1}, t\right)\right)-g\left(x_{1}, t\right)\left(h_{2}^{\prime \prime}(t)-f\left(x_{2}, t\right)\right)\right\|_{C[0, T]}+\right.$ 


$$
\begin{aligned}
& +\left(\sum_{k=1}^{\infty} \lambda_{k}^{-2}\right)^{\frac{1}{2}}\left\|\left|g\left(x_{1}, t\right)\right|+\mid g\left(x_{2}, t\right)\right\|_{C[0, T]}\left[\sum_{i=1}^{2}\left(\sum_{k=1}^{\infty}\left(\lambda_{k}^{3}\left|\varphi_{i k}\right|\right)^{2}\right)^{\frac{1}{2}}+(1+\delta)\left[\sum_{i=1}^{2}\left(\sum_{k=1}^{\infty}\left(\lambda_{k}^{3}\left|\psi_{i k}\right|\right)^{2}\right)^{\frac{1}{2}}+\right.\right. \\
& \left.+T\|p(t)\|_{C[0, T]} \sum_{i=1}^{2}\left(\sum_{k=1}^{\infty}\left(\lambda_{k}^{3}\left\|u_{i k}(t)\right\|_{C[0, T]}\right)^{2}\right)^{\frac{1}{2}}\right]+(1+\delta)\left[\sqrt{T} \sum_{i=1}^{2}\left(\sum_{0}^{T} \sum_{k=1}^{\infty}\left(\lambda_{k}^{3}\left|f_{i k}(\tau)\right|\right)^{2} d \tau\right)^{\frac{1}{2}}+\right. \\
& \left.\left.\left.+T\|a(t)\|_{C[0, T]} \sum_{i=1}^{2}\left(\sum_{k=1}^{\infty}\left(\lambda_{k}^{3}\left\|u_{i k}(t)\right\|_{C[0, T]}\right)^{2}\right)^{\frac{1}{2}}+\sqrt{T} \sum_{i=1}^{2}\left(\int_{0}^{T} \sum_{k=1}^{\infty}\left(\lambda_{k}^{3}\left|g_{i k}(\tau)\right|\right)^{2} d \tau\right)^{\frac{1}{2}}\|b(t)\|_{C[0, T]}\right]\right]\right\},
\end{aligned}
$$

(26)

$$
\begin{aligned}
& \|\tilde{b}(t)\|_{C[0, T]} \leq\left\|[h(t)]^{-1}\right\|_{C[0, T]}\left\{h_{1}(t)\left(h_{2}^{\prime \prime}(t)-f\left(x_{2}, t\right)\right)-h_{2}(t)\left(h_{1}^{\prime \prime}(t)-f\left(x_{1}, t\right)\right) \|_{C[0, T]}+\right. \\
& +\left(\sum_{k=1}^{\infty} \lambda_{k}^{-2}\right)^{\frac{1}{2}}\left\|\left|h_{1}(t)\right|+\mid h_{2}(t)\right\|_{C[0, T]}\left[\sum_{i=1}^{2}\left(\sum_{k=1}^{\infty}\left(\lambda_{k}^{3}\left|\varphi_{i k}\right|\right)^{2}\right)^{\frac{1}{2}}+(1+\delta)\left[\sum_{i=1}^{2}\left(\sum_{k=1}^{\infty}\left(\lambda_{k}^{3}\left|\psi_{i k}\right|\right)^{2}\right)^{\frac{1}{2}}+\right.\right. \\
& \left.+T\|p(t)\|_{C[0, T]} \sum_{i=1}^{2}\left(\sum_{k=1}^{\infty}\left(\lambda_{k}^{3}\left\|u_{i k}(t)\right\|_{C[0, T]}\right)^{2}\right)^{\frac{1}{2}}\right]+(1+\delta)\left[\sqrt{T} \sum_{i=1}^{2}\left(\int_{0}^{T} \sum_{k=1}^{\infty}\left(\lambda_{k}^{3}\left|f_{i k}(\tau)\right|\right)^{2} d \tau\right)^{\frac{1}{2}}+\right. \\
& \left.\left.+T\|a(t)\|_{C[0, T]} \sum_{i=1}^{2}\left(\sum_{k=1}^{\infty}\left(\lambda_{k}^{3}\left\|u_{i k}(t)\right\|_{C[0, T]}\right)^{2}\right)^{\frac{1}{2}}+\sqrt{T} \sum_{i=1}^{2}\left(\sum_{0}^{T} \sum_{k=1}^{\infty}\left(\lambda_{k}^{3}\left|g_{i k}(\tau)\right|\right)^{2} d \tau\right)^{\frac{1}{2}}\|b(t)\|_{C[0, T]}\right]\right]
\end{aligned}
$$

Suppose that the data of the problem (1)-(3), (6), (7) satisfy the following condition

$Q_{1} . \varphi(x) \in C^{2}[0,1], \varphi^{\prime \prime \prime}(x) \in L_{2}(0,1), \varphi(0)=\varphi(1), \varphi^{\prime}(0)=\varphi^{\prime}(1), \varphi^{\prime \prime}(0)=\varphi^{\prime \prime}(1)$.

$Q_{2} \cdot \psi(x) \in C^{2}[0,1], \psi^{\prime \prime \prime}(x) \in L_{2}(0,1), \psi(0)=\psi(1), \psi^{\prime}(0)=\psi^{\prime}(1), \psi^{\prime \prime}(0)=\psi^{\prime \prime}(1)$.

$Q_{3} \cdot f(x, t), f_{x}(x, t), f_{x x}(x, t) \in C\left(D_{T}\right), f_{x x x}(x, t) \in L_{2}\left(D_{T}\right)$,

$f(0, t)=f(1, t), f_{x}(0, t)=f_{x}(1, t), f_{x x}(0, t)=f_{x x}(1, t)(0 \leq t \leq T)$.

$Q_{4} \cdot g(x, t), g_{x}(x, t), g_{x x}(x, t) \in C\left(D_{T}\right), g_{x x x}(x, t) \in L_{2}\left(D_{T}\right)$, 
$g(0, t)=g(1, t), g_{x}(0, t)=g_{x}(1, t), g_{x x}(0, t)=g_{x x}(1, t)(0 \leq t \leq T)$.

$Q_{5} . \delta \geq 0, p(t) \in C[0, T], h(t) \in C^{2}[0, T], h(t) \neq 0(0 \leq t \leq T)$.

Then, from (25)-(27), we get

$$
\begin{aligned}
& \|\tilde{u}(x, t)\|_{B_{2, T}^{3}} \leq A_{1}(T)+B_{1}(T)\|a(t)\|_{C[0, T]}\|u(x, t)\|_{B_{2, T}^{3}}+C_{1}(T)\|u(x, t)\|_{B_{2, T}^{3}}+D_{1}(T)\|b(t)\|_{C[0, T]}, \\
& \|\tilde{a}(t)\|_{C[0, T]} \leq A_{2}(T)+B_{2}(T)\|a(t)\|_{C[0, T]}\|u(x, t)\|_{B_{2, T}^{3}}+C_{2}(T)\|u(x, t)\|_{B_{2, T}^{3}}+D_{2}(T)\|b(t)\|_{C[0, T]},
\end{aligned}
$$

$\|\tilde{b}(t)\|_{C[0, T]} \leq A_{3}(T)+B_{3}(T)\|a(t)\|_{C[0, T]}\|u(x, t)\|_{B_{2, T}^{3}}+C_{3}(T)\|u(x, t)\|_{B_{2, T}^{3}}+D_{3}(T)\|b(t)\|_{C[0, T]}$,

where

$$
\begin{aligned}
& A_{1}(T)=\|\varphi(x)\|_{L_{2}(0,1)}+T\|\psi(x)\|_{L_{2}(0,1)}+(T+\delta) \sqrt{T}\|f(x, t)\|_{L_{2}\left(D_{T}\right)}+ \\
& +\sqrt{6}\left\|\varphi^{\prime \prime \prime}(x)\right\|_{L_{2}(0,1)}+\sqrt{6}(1+\delta)\left\|\psi^{\prime \prime \prime}(x)\right\|_{L_{2}(0,1)}+(1+\delta) \sqrt{6 T}\left\|f_{x x x}(x, t)\right\|_{L_{2}\left(D_{T}\right)}, \\
& B_{1}(T)=T(T+\delta)+\sqrt{6}(1+\delta) T, \\
& C_{1}(T)=T\|p(t)\|_{C[0, T]}(T+\sqrt{6}(1+\delta)) \\
& D_{1}(T)=(T+\delta) \sqrt{T}\|g(x, t)\|_{L_{2}\left(D_{T}\right)}+(1+\delta) \sqrt{6 T}\left\|g_{x x x}(x, t)\right\|_{L_{2}\left(D_{T}\right)}, \\
& A_{2}(T)=\left\|[h(t)]^{-1}\right\|_{C[0, T]}\left\{\mid g\left(x_{1}, t\right)\left(h_{1}^{\prime \prime}(t)-f\left(x_{1}, t\right)\right)-g\left(x_{1}, t\right)\left(h_{2}^{\prime \prime}(t)-f\left(x_{2}, t\right)\right) \|_{C[0, T]}+\right. \\
& +\left(\sum_{k=1}^{\infty} \lambda_{k}^{-2}\right)^{\frac{1}{2}}\left\|\left|g\left(x_{1}, t\right)\right|+\mid g\left(x_{2}, t\right)\right\| \|_{C[0, T]}\left(2\left\|\varphi^{\prime \prime \prime}(x)\right\|_{L_{2}(0,1)}+2(1+\delta)\left\|\psi^{\prime \prime \prime}(x)\right\|_{L_{2}(0,1)}+\right. \\
& \left.\left.+2(1+\delta) \sqrt{T}\left\|f_{x x x}(x, t)\right\|_{L_{2}\left(D_{T}\right)}\right)\right\} \\
& B_{2}(T)=\left\|[h(t)]^{-1}\right\|_{C[0, T]}\left(\sum_{k=1}^{\infty} \lambda_{k}^{-2}\right)^{\frac{1}{2}}\left\|\left|g\left(x_{1}, t\right)\right|+\mid g\left(x_{2}, t\right)\right\|_{C[0, T]}(1+\delta) T,
\end{aligned}
$$




$$
\begin{gathered}
C_{2}(T)=\left\|[h(t)]^{-1}\right\|_{C[0, T]}\left(\sum_{k=1}^{\infty} \lambda_{k}^{-2}\right)^{\frac{1}{2}}\left\|\left|g\left(x_{1}, t\right)\right|+\mid g\left(x_{2}, t\right)\right\|_{C[0, T]}(1+\delta)\|p(t)\|_{C[0, T]} T, \\
D_{2}(T)=2\left\|[h(t)]^{-1}\right\|_{C[0, T]}\left(\sum_{k=1}^{\infty} \lambda_{k}^{-2}\right)^{\frac{1}{2}}\left\|\left|g\left(x_{1}, t\right)\right|+\mid g\left(x_{2}, t\right)\right\|_{C[0, T]}(1+\delta) \sqrt{T}\left\|g_{x x x}(x, t)\right\|_{L_{2}\left(D_{T}\right)}, \\
A_{3}(T)=\left\|[h(t)]^{-1}\right\|_{C[0, T]}\left\{\mid h_{1}(t)\left(h_{2}^{\prime \prime}(t)-f\left(x_{2}, t\right)\right)-h_{2}(t)\left(h_{1}^{\prime \prime}(t)-f\left(x_{1}, t\right)\right) \|_{C[0, T]}+\right. \\
+\left(\sum_{k=1}^{\infty} \lambda_{k}^{-2}\right)^{\frac{1}{2}}\left\|\left|h_{1}(t)\right|+\mid h_{2}(t)\right\| \|_{C[0, T]}\left(2\left\|\varphi^{\prime \prime \prime}(x)\right\|_{L_{2}(0,1)}+2(1+\delta)\left\|\psi^{\prime \prime \prime}(x)\right\|_{L_{2}(0,1)}+\right. \\
\left.\left.+2(1+\delta) \sqrt{T}\left\|f_{x x x}(x, t)\right\|_{L_{2}\left(D_{T}\right)}\right)\right\}, \\
D_{2}(T)=2\left\|[h(t)]^{-1}\right\|_{C[0, T]}\left(\sum_{k=1}^{\infty} \lambda_{k}^{-2}\right)^{\frac{1}{2}}\left\|\left|g\left(x_{1}, t\right)\right|+\mid g\left(x_{2}, t\right)\right\|\left\|_{C[0, T]}(1+\delta) \sqrt{T}\right\| g_{x x x}(x, t) \|_{L_{2}\left(D_{T}\right)}, \\
C_{2}(T)=\left\|[h(t)]^{-1}\right\|_{C[0, T]}\left(\sum_{k=1}^{\infty} \lambda_{k}^{-2}\right)^{\frac{1}{2}}\left\|\left|h_{1}(t)\right|+\mid h_{2}(t)\right\|_{C[0, T]}(1+\delta)\|p(t)\|_{C[0, T]} T, \\
B_{2}(T)=\left\|[h(t)]^{-1}\right\|\left\|_{C[0, T]}\left(\sum_{k=1}^{\infty} \lambda_{k}^{-2}\right)^{\frac{1}{2}}\right\|\left|h_{1}(t)\right|+\mid h_{2}(t) \|_{C[0, T]}(1+\delta) T,
\end{gathered}
$$

It follows from the inequalities (28)-(30) that.

$$
\begin{aligned}
& \|\tilde{u}(x, t)\|_{B_{2, T}^{3}}+\|\tilde{a}(t)\|_{C[0, T]}+\|\tilde{b}(t)\|_{C[0, T]} \leq \\
& \quad \leq A(T)+B(T)\|a(t)\|_{C[0, T]}\|u(x, t)\|_{B_{2, T}^{3}}+C(T)\|u(x, t)\|_{B_{2, T}^{3}}+D(T)\|b(t)\|_{C[0, T]},
\end{aligned}
$$

where

$$
\begin{aligned}
& A(T)=A_{1}(T)+A_{2}(T)+A_{3}(T), \quad B(T)=B_{1}(T)+B_{2}(T)+B_{3}(T), \\
& C(T)=C_{1}(T)+C_{2}(T)+C_{3}(T), \quad D(T)=D_{1}(T)+D_{2}(T)+D_{3}(T) .
\end{aligned}
$$

So, we can prove the following theorem:

Theorem 1. Let conditions $Q_{1}-Q_{5}$ be satisfied, and 


$$
(B(T)(A(T)+2)+C(T)+D(T))(A(T)+2)<1 .
$$

Then problem (1)-(3), (6), (7) has a unique solution in the sphere $K=K_{R}\left(\|z\|_{E_{T}^{3}} \leq R=A(T)+2\right)$ of the space $E_{T}^{3}$.

Proof. In the space $E_{T}^{3}$ consider the equation

$$
z=\Phi z
$$

where $z=\{u, a, b\}$, , the components $\Phi_{i}(u, a, b)(i=1,2,3)$ of the operator $\Phi(u, a, b)$ are determined by the right hand sides of equations (19), (22) and (23). Consider the operator $\Phi(u, a, b)$ in the sphere $K=K_{R}$ from $E_{T}^{3}$. Similar to (31), we get that for any $z_{1}, z_{2}, z_{3} \in K_{R}$ the following estimations are valid:

$$
\begin{aligned}
\|\Phi z\|_{E_{T}^{3}} \leq A(T)+ & B(T)\|a(t)\|_{C[0, T]}\|u(x, t)\|_{B_{2, T}^{3}}+C(T)\|u(x, t)\|_{B_{2, T}^{3}}+D(T)\|b(t)\|_{C[0, T]} \leq \\
\leq & A(T)+B(T)(A(T)+2)^{2}+C(T)(A(T)+2)+D(T)(A(T)+2), \\
\left\|\Phi z_{1}-\Phi z_{2}\right\|_{E_{T}^{3}} \leq & B(T) R\left(\left\|a_{1}(t)-a_{2}(t)\right\|_{C[0, T]}+\left\|u_{1}(x, t)-u_{2}(x, t)\right\|_{B_{2, T}^{3}}\right)+ \\
+ & C(T)\left\|u_{1}(x, t)-u_{2}(x, t)\right\|_{B_{2, T}^{3}}+D(T)\left\|b_{1}(t)-b_{2}(t)\right\|_{C[0, T]},
\end{aligned}
$$

Then, it follows from (32 together with the estimates (34) and (35) that the operator $\Phi$ acts in the ball $K=K_{R}$ and is contractive. Therefore, in the ball $K=K_{R}$ the operator $\Phi$ has a unique fixed point $\{z\}=\{u, a, b\}$, that is a unique solution to the equation (33), i.e. a unique solution to the system (19),(22), (23).

The function $u(x, t)$, as an element of the space $B_{2, T}^{3}$, is continuous and has continuous derivatives $u_{x}(x, t), u_{x x}(x, t)$ in $D_{T}$.

Now, from (13) and (14) we get

$$
\begin{gathered}
\left\|u_{10}^{\prime \prime}(t)\right\|_{C[0, T]} \leq\|a(t)\|_{C[0, T]}\left\|u_{10}(t)\right\|_{C[0, T]}+\|b(t)\|_{C[0, T]}\|g(x, t)\|_{C[0, T]}\left\|_{L_{2}(0,1)}+\right\|\|f(x, t)\|_{C[0, T]} \|_{L_{2}(0,1)}, \\
\left(\sum_{k=1}^{\infty}\left(\lambda_{i k}\left\|u_{i k}(t)\right\|_{C[0, T]}\right)^{2}\right)^{\frac{1}{2}} \leq \sqrt{2}\left(\sum_{k=1}^{\infty}\left(\lambda_{k}^{3}\left\|u_{i k}(t)\right\|_{C[0, T]}\right)^{2}\right)^{\frac{1}{2}}+ \\
+\sqrt{2}\|\| a(t) u_{x}(x, t)+b(t) g_{x}(x, t)+f_{x}(x, t)\left\|_{C[0, T]}\right\|_{L_{2}(0,1)}
\end{gathered}
$$


Hence it follows that $u_{t t}(x, t)$ is continuous in $D_{T}$. It is easy to verify that equation (1) and conditions (2), (3),(6) and (7) are satisfied in the ordinary sense.

Consequently, $\{u(x, t), a(t), b(t)\}$ is a solution of problem (1)-(3), (6), (.7), and by Lemma 2 it is unique in the bal $K=K_{R}$. The theorem is proved..

By Lemma 1 the unique solvability of the initial problem (1)-(5) follows from the theorem.

Theorem 2. Let all the conditions of Theorem 1 be fulfilled and

$$
\begin{gathered}
\int_{0}^{1} f(x, t) d x=0, \int_{0}^{1} g(x, t) d x=0(0 \leq t \leq T), \\
\int_{0}^{1} \varphi(x) d x=0, \int_{0}^{1} \psi(x) d x=0, \\
\varphi\left(x_{i}\right)=h_{i}(0)-\delta h_{i}^{\prime}(0), \psi\left(x_{i}\right)=h_{i}^{\prime}(T)-\int_{0}^{T} p(t) h_{i}(t) d t(i=1,2), \\
T\left((2 T+\delta)(A(T)+2)+(T+\delta)\|p(t)\|_{C[0, T]}\right)<1 .
\end{gathered}
$$

Then the problem (1)-(5) has a unique classical solution in the ball $K=K_{R}\left(\|z\|_{E_{T}^{3}} \leq A(T)+2\right)$ of the space $E_{T}^{3}$.

\section{REFERENCES}

[1] Tikhonov A.N., On stability of inverse problems, Dokl. AN SSSR, 39-5 (1943) 195198 (in Russian).

[2] Lavrent'ev M.M., On an inverse problem for a wave equation, Dokl. AN SSSR, 157-3 (1964) 520-521 (in Russian).

[3] Lavrent'ev M.M., Romanov V.G., Shishatsky S.T. Ill-posed problems of mathematical physics and analysis, M., Nauka, 1980 (in Russian).

[4] Ivanov V.K., Vasin V.V., Tanana V.P., Theory of linear ill-posed problems and its applications, M., Nauka, 1978 (in Russian).
[5] Denisov A.M., Introduction to theory of inverse problems, M., MGU, 1994 (in Russian).

[6] Mehraliyev Ya.T., On solvability of an inverse boundary value problem for a second order elliptic equation, Vest. Tverskogo Gos. Univ., Ser. Prikladnaya Matematika, 23 (2011) 25-38 (in Russian).

[7] Budak B.M., Samarskii A.A.,.Tikhonov A.N, A Collection of Problems in Mathematical Physics, M. Nauka, 1972 (in Russian). 